\title{
Minimum Energy Data Gathering in Correlated Sensor Networks with Cooperative Transmission
}

\author{
Laxminarayana S Pillutla and Vikram Krishnamurthy \\ Department of Electrical and Computer Engineering \\ The University of British Columbia \\ 2332 Main Mall, Vancouver, BC V6T1Z4, Canada. \\ E-mail:\{laxp, vikramk\}@ece.ubc.ca
}

\begin{abstract}
We consider combination of Distributed Source Coding (DSC) and cooperative transmission techniques to improve energy efficiency in sensor networks. To start with we formulate the data gathering problem in correlated wireless sensor networks with cooperative Multiple Input and Multiple Output (MIMO) transmission at the physical layer and DSC at the application layer. Using the concepts of super and sub modularity on a lattice, we analytically quantify as how the optimal constellation size and the optimal number of cooperating nodes vary with respect to the correlation coefficient. In particular, we show that the optimal constellation size is an increasing function of the correlation coefficient. For the MIMO transmission case, the optimal number of cooperating nodes is a decreasing function of the correlation coefficient. We also prove that in a MIMO transmission based scheme the optimal constellation size adopted by each cooperating node is a decreasing function of the number of cooperating nodes. Also, it is shown that the optimal number of cooperating nodes is a decreasing function of the constellation size adopted by each cooperating node. Finally through our numerical results, it is shown that significant energy savings can be obtained if correlation in the network is exploited. Also, when the desired probability of error is small MIMO transmission can lead to large scale energy savings.
\end{abstract}

\section{INTRODUCTION}

A wireless sensor network (WSN) consists of large number of spatially distributed devices called nodes which cooperate to accomplish various high level tasks. An important feature of WSN is high degree of correlation in the data sensed by nodes, owing to the high node density. This correlation among the data sensed by nodes can be exploited to minimize the amount of data transmitted by nodes, hence the total energy consumption of WSN. Techniques based on distributed source coding (DSC) can be used to accomplish this.

To this end, in [1], the authors consider DSC and routing in correlated sensor networks. In this case, the joint problem of optimizing transmission structure and source coding becomes decoupled. The advantage of this method lies in determining optimal transmission structure in a simple manner; however the coding becomes complex owing to the need for global network knowledge and correlation structure.

To overcome the problem of knowing correlation a-priori, in [2], the authors consider another approach called explicit communication wherein nodes can exploit correlation by receiving explicit side information from other nodes, which is possible when other nodes use a node as relay. This approach leads to simple source coding, but the transmission structure optimization is hard.

\section{A. Main Results}

We consider combination of cooperative multiple input and multiple output (MIMO) and DSC techniques to improve energy efficiency of WSN. The problem of minimum energy data gathering $(M E D G)$ in WSN based on cooperative multiple input and multiple output (MIMO) techniques was considered in [3], although correlation in data sensed by nodes was not considered. We also study as how the correlation coefficient, which is an application layer parameter, impacts the physical layer design variables such as constellation size and number of cooperating nodes. To obtain these structural results, we use concepts in monotone comparative statics [4], which are extensively used in economics literature, to study as how an optimal solution to a parametrized optimization problem varies with respect to the parameter.

Our main results ${ }^{1}$ are summarized as follows:

1) We prove that the optimal constellation size adopted by a sensor to minimize total energy consumption in the network is an increasing function of the correlation coefficient. This implies that for the case when data is highly correlated to minimize the energy consumption, each sensor adopts higher constellation size.

2) For the case of cooperative transmission based on MIMO techniques we prove that the optimal number of cooperating nodes to minimize total energy consumption in the network is a decreasing function of the correlation coefficient.

3) Also for a cooperative MIMO transmission scheme we prove that the optimal constellation size adopted by each cooperating node to minimize energy consumption decreases with increase in number of cooperating nodes. The reverse implication is also true i.e. the optimal number of cooperating nodes to minimize energy consumption decreases with increase in the constellation size.

\footnotetext{
${ }^{1}$ A complete version of this paper has been submitted to IEEE Trans. on Signal Processing [5]
} 
4) Finally from our numerical results it has been observed that significant energy savings can be obtained by exploiting correlation in the network. For moderate values of probability of error, when circuit energy consumption is taken into account SISO transmission can be more energy efficient than MIMO transmission. However, when circuit energy consumption is ignored then MIMO transmission can be more energy efficient. Also when circuit energy consumption is taken into account then shortest path routing may not be optimal. For the case when desired probability of error is very small MIMO transmission is more energy efficient.

\section{B. Paper Organization}

The rest of this paper is organized as follows: Section II contains system model description and assumptions. Section III contains formulation of MEDG in a correlated WSN. To make the paper self-contained we include a brief survey on monotone comparative statics in Section IV. Section V contains structural results on optimal constellation size and number of cooperating nodes. Section VI contains numerical results and Section VII contains concluding remarks.

\section{Wireless Sensor Network Model And ASSUMPTIONS}

We consider data gathering using WSN. For the purpose of our analysis we assume signal along the route to the destination forms a one dimensional stationary Gauss-Markov process. This is valid in the case of acoustic sensor networks where multiple acoustic sensor nodes deployed receive time delayed version of the same signal, and hence it is reasonable to assume that the signal sensed by successive stages of the network is correlated. The Gauss-Markov assumption on the underlying signal process leads us to the following state-space representation of the signal process

$$
\begin{aligned}
x_{k} & =a_{k-1} x_{k-1}+u_{k-1}, \text { for } k=1,2, \cdots, I, \\
u_{k-1} & \sim \mathcal{N}\left(0, P_{0}\left(1-a_{k-1}^{2}\right)\right),
\end{aligned}
$$

where $a_{k-1}$ denotes the correlation between $(k-1)^{\text {th }}$ and $k^{\text {th }}$ stages of the network. A natural model for correlation is the Power Exponential Model. In this model $a_{k}$ is modeled as $a_{k-1}=e^{-A d_{k-1}}$, where $A$ is the field diffusion coefficient and $d_{k-1}$ denotes the distance between $(k-1)^{\text {th }}$ and $k^{\text {th }}$ stage of the network.

Each stage of the network on way to the destination observes a noisy version of the process i.e.

$$
y_{k}=x_{k}+n_{k}, n_{k} \sim \mathcal{N}\left(0, \sigma^{2}\right),
$$

where $\sigma^{2}$ denotes the variance of observation noise. The observation SNR at each stage of the network is defined as $\Gamma=\frac{P_{0}}{\sigma^{2}}$.

We abstract the WSN as a directed graph $G=(V, E)$ where $V$ denotes the set of stages and $E$ denotes the set of directed links. We consider the case of single sink data gathering only. We assume there are $(I+1)$ stages in the network, where $1,2, \cdots, I$ denote the data gathering stages and the $(I+1)^{\text {th }}$ stage denotes the sink node (or destination node). Every directed link $e_{i j} \in E$ that connects two stages $(i, j) \in V$ has a cost $\left(E_{i j}\right)$ associated with it. For a given constellation size $b_{i j}$ on the link, the cost $E_{i j}$ depends on square of the distance between the nodes and the circuit power consumption.

Each stage of the network contain one node (for SISO transmission case) or more than one node (for MIMO transmission case). The transmission in SISO case is straightforward, while in MIMO case a node called the sensing node makes an observation of the underlying process, quantizes and broadcasts locally to other nodes in that stage for cooperative transmission. Upon local information circulation with in each stage, the nodes transmit cooperatively the data originated at its own stage along with the data received from previous stages to the next stage. The next stage for both the cases is determined by solution to the energy optimization problem formulated in Section III. The cooperation among the nodes is accomplished based on the well-known space-time block codes (STBC) [6].

Since for short range communications circuit energy can be significant, hence we consider circuit energy consumption along with the transmission energy consumption as in [7] and [3]. The antenna of a sensor node is typically at the ground level, hence we model the channel between sensors to be of Rayleigh fading type. We assume each sensor node employs $\mathrm{M}$-ary quadrature amplitude modulation (M-QAM) for digital modulation. We assume each sensor performs lossless source coding. Since the distance between nodes in each stage of the network is small compared to the distance between two stages of the network, hence we assume all the nodes at a given stage to be equidistant from the neighboring stage.

\section{Minimum Energy Data Gathering (MEDG) In CORRELATED SENSOR NETWORKS}

In this section, we formulate the problem of MEDG in a WSN, for the linear Gauss-Markov state-space model assumed in equations (1) and (2).

\section{A. Expression for Total Energy Consumption per bit using SISO Techniques}

The total power consumed in a sensor node employing QAM while transmitting over link $(i, j)$ is given as [7]

$$
\begin{aligned}
& P_{S I S O}^{i j}=\underbrace{\frac{1}{6}(1+\alpha)\left(\frac{1}{p_{b}}\right)\left(2^{b_{i j}}-1\right) N_{0} G_{0} d_{i j}^{2} B}_{\text {Transmission power }}+ \\
& \underbrace{\left(P_{T}+P_{R}\right)}_{\text {Circuit power }}\left(\text { for } b_{i j} \geq 2\right),
\end{aligned}
$$

where $\alpha$ is a constant defined by the power amplifier efficiency, $p_{b}$ is the desired probability of error, $b_{i j}$ denotes the constellation size adopted on link $(i, j), N_{0}$ is the noise spectral density, $G_{0}$ is the attenuation factor, $d_{i j}$ is length of the link $(i, j), B$ is the symbol rate and $P_{T}, P_{R}$ denote the transmitter and receiver circuit power consumption. 
Thus the energy consumption per bit in transmitting over link $(i, j)$ using SISO transmission can be written as

$$
\begin{aligned}
E_{S I S O}^{i j}= & \left(\frac{P_{S I S O}^{i j}}{B}\right) \frac{1}{b_{i j}}, \\
= & \frac{1}{6}(1+\alpha)\left(\frac{1}{p_{b}}\right)\left(\frac{2^{b_{i j}}-1}{b_{i j}}\right) N_{0} G_{0} d_{i j}^{2}+ \\
& \frac{\left(P_{T}+P_{R}\right)}{B} \frac{1}{b_{i j}}\left(\text { for } b_{i j} \geq 2\right) .
\end{aligned}
$$

B. Expression for Total Energy Consumption per bit using MIMO Techniques

As mentioned in Section II, for cooperative transmission to be possible there needs to be local information circulation. To accomplish this, we assume a node in each stage of the network makes an observation, encodes at rate $R_{k}$ (as determined in next subsection) and broadcasts to the other $n_{k}-1$ nodes in that stage for cooperative transmission.

The energy consumption for this local broadcast in $k^{\text {th }}$ stage can be written as in Equation (4) to be

$$
\begin{aligned}
& E_{\text {local }}^{k}=\frac{1}{6}(1+\alpha)\left(\frac{1}{p_{b}}\right)\left(\frac{2^{b_{\text {local }}}-1}{b_{\text {local }}}\right) G_{0} N_{0} d_{\max , k}^{2}+ \\
& \left(n_{k}-1\right) \frac{\left(P_{T}+P_{R}\right)}{B b_{\text {local }}}
\end{aligned}
$$

where $b_{\text {local }}$ denotes the constellation size adopted for local transmission and $d_{\max , k}$ denotes the maximum transmission radius of stage $k$. The factor of $\left(n_{k}-1\right)$ in the second term of Equation (5) is due to the fact that during local broadcast there are always $\left(n_{k}-1\right)$ circuits listening.

For the purpose of our analysis and simulation in this paper we assume $b_{\text {local }}$ as fixed. Consequently, the energy consumption for local broadcast at the $k^{\text {th }}$ stage $\left(E_{\text {local }}^{k}\right)$ is a function of only the number of cooperating nodes $n_{k}$ present in the $k^{\text {th }}$ stage.

If we assume $n$ nodes in a cluster cooperate and transmit then the energy consumption per bit $\left(E_{b}\right)$ for cooperative transmission can be upper bound as [7]

$$
E_{b} \leq \frac{2}{3} n\left(\frac{4}{p_{b}}\right)^{\frac{1}{n}}\left(\frac{2^{b}-1}{b^{\frac{1}{n}}+1}\right) N_{0}(\text { for } b \geq 2),
$$

where $b$ is the constellation size adopted for cooperative transmission, $p_{b}$ is the desired probability of error and $N_{0}$ is the noise spectral density.

Approximating the energy consumption per bit in Equation (6) as an equality, we can express the total power consumed for cooperative transmission over the link $(i, j)$ as

$$
P_{L H}^{i j}=\underbrace{(1+\alpha) E_{b} b_{i j} G_{0} d_{i j}^{2} B}_{\text {Transmission Power }}+\underbrace{n_{i}\left(P_{T}+P_{R}\right)}_{\text {Circuit Power }},
$$

where $E_{b}$ is given by Equation (6).

Thus, the total energy consumption per bit for transmission over the link $(i, j)$ with MIMO transmission can be written as

$$
E_{M I M O}^{i j}=\left(\frac{P_{L H}^{i j}}{B_{e}}\right) \frac{1}{b_{i j}}+E_{\text {local }}^{i},
$$

where $\left(B_{e}\right)$ is the effective bandwidth and is equal to $(B r)$, $(r)$ is the spatial rate of STBC employed and $\left(E_{\text {local }}^{i}\right)$ is given by Equation (5).

For the general case of $n$ cooperating nodes as observed in [6], it may not be possible to design $r=1$ STBC for complex constellations such as QAM or phase shift keying (PSK). Consequently, $r$ in our formulation depends on number of cooperating nodes $n$.

Approximating Equation (6) as an equality we can express Equation (8) as

$$
\begin{aligned}
& E_{M I M O}^{i j}=\frac{2}{3}(1+\alpha) n_{i}\left(\frac{4}{p_{b}}\right)^{\frac{1}{n_{i}}}\left(\frac{2^{b_{i j}}-1}{b_{i j}^{\frac{1}{n_{i}}}+1}\right) N_{0} G_{0} d_{i j}^{2} \\
& \left(\frac{1}{r}\right)+n_{i} \frac{\left(P_{T}+P_{R}\right)}{B r}\left(\frac{1}{b_{i j}}\right)+E_{T x-\text { local }}+ \\
& \left(n_{i}-1\right) \frac{\left(P_{T}+P_{R}\right)}{B b_{\text {local }}}
\end{aligned}
$$

where $E_{T x-\text { local }}$ is given by first term in Equation (5).

A close look at Equation (9) reveals that when a $r \leq 1$ STBC is used then it can lead to increased energy consumption.

\section{Formulation of MEDG in Correlated WSN}

In this subsection we formulate the problem of minimum energy data gathering (MEDG) in correlated sensor networks.

In a WSN since energy consumption needs to be minimized to enhance the lifetime of the network, hence the optimization problem to minimize energy consumption can be stated as follows:

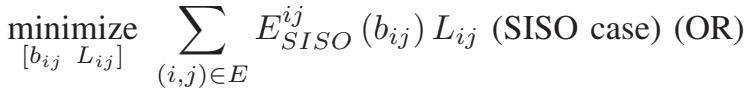

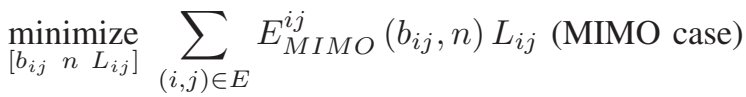

$$
\begin{aligned}
& \sum_{j:(i, j) \in E} L_{i j}-\sum_{j:(j, i) \in E} L_{j i}=R_{i} \text { for } i=1,2,3, \cdots, I+1 \\
& 2 \leq b_{i j} \leq b_{\max }^{i j} \\
& 1 \leq n \leq N
\end{aligned}
$$

where $L_{i j}$ denotes the flow (in terms of bits) associated with the link $(i, j)$. The constraint in Equation (11) is the flow conservation constraint which ensures at any stage the difference between outgoing flow and the incoming flow equals the data generated at the corresponding stage. In Equation (12) $b_{\max }^{i j}$ is determined so that the sensor node does not transmit beyond its peak power.

The rate allocations $R_{k}$ in Equation (11) are determined so that the rate admissibility constraint is satisfied. For a network with single sink the rate admissibility constraint can be written as

$$
\sum_{k=1}^{I} R_{k} \geq H\left(y_{1}, y_{2}, \cdots, y_{I}\right)
$$


where $H\left(z_{1}, z_{2}, \cdots, z_{K}\right)$ denotes entropy of $z_{1}, z_{2}, \cdots, z_{K}$.

In the presence of knowledge about correlation between various stages of the network, we can choose individual $R_{k}$ as [1]

$R_{k}=H\left(y_{k} \mid y_{k+1}, y_{k+2}, \cdots, y_{I}\right)$ for $k=1,2, \cdots, I-1$,

$R_{I}=H\left(y_{I}\right)$,

where $H\left(z_{k} \mid z_{k+1}, z_{k+2}, \cdots, z_{I}\right)$ denotes the conditional entropy of $z_{k}$ given $z_{k+1}, z_{k+2}, \cdots, z_{I}$ and $H\left(z_{I}\right)$ denotes the entropy of $z_{I}$.

Approximating the discrete entropy using the differential entropy we can compute equations (15) and (16) for the GaussMarkov model assumed in equations (1) and (2) as

$$
\begin{aligned}
R_{k}= & \frac{1}{2} \log _{2}\left(\frac{2 \pi e \sigma^{2}}{\Delta^{2}}\right)\left(1+\frac{\Sigma_{k \mid k-1}}{\sigma^{2}}\right), \\
& \text { for } k=1,2, \cdots, I-1, \\
R_{I}= & \frac{1}{2} \log _{2}\left(\frac{2 \pi e \sigma^{2}}{\Delta^{2}}\right)(\Gamma+1),
\end{aligned}
$$

where $\Sigma_{k \mid k-1}$ is the predictor covariance, $\Delta$ is the quantization stepsize, $\Gamma$ is the observation SNR, and $\sigma^{2}$ is the observation noise variance.

For the case of stage 1 we choose $\Sigma_{1 \mid 0}=P_{0}$, hence its encoding rate $R_{1}=\frac{1}{2} \log _{2}\left(\frac{2 \pi e \sigma^{2}}{\Delta^{2}}\right)(\Gamma+1)$. Using the Kalman filter recursion we can upper bound $R_{k}$ for $k=$ $2,3 \cdots, I-1[8]$ as

$$
R_{k} \leq \frac{1}{2} \log _{2}\left(\frac{2 \pi e \sigma^{2}}{\Delta^{2}}\right)\left((\Gamma+1)-a_{k-1}^{2}(\Gamma-1)\right),
$$

for $k=2,3, \cdots, I-1$.For the rest of our analysis in this paper we assume that the sensing node in stage 1 encodes at rate $R_{1}$, stages $k=2,3, \cdots, I-1$ encode at a rate equal to the upper bound in Equation (19), and the sensing node in stage $I$ encodes at rate $R_{I}$ given by Equation (18).

\section{Structural Results on Optimal Constellation SizE AND Number OF COOPERATING NODES}

For the purpose of analysis in this section we assume a homogeneous network so that the distance (or spacing) between consecutive stages of the network is same i.e. $d_{i j}=d$. Consequently the correlation in data sensed by successive stages is same i.e. $a_{k}=a$. We also assume that every stage of the network employs same constellation size i.e. $b_{i j}=b$. Finally, we also assume that the routing is fixed such that each stage of the network transmits to an immediate downstream stage.

We omit the proofs of structural results in this paper. Interested readers are referred to [5] for details.

\section{A. Structural Result on Optimal Constellation Size ( $\left.b^{*}\right)$ versus correlation coefficient $(a)$}

Since the $k^{\text {th }}$ stage (for $k=1,2, \cdots, I$ ) in the network adopts rate $R$ given in Equation (19), while the $I^{\text {th }}$ stage adopts rate $R_{I}$ given in Equation (18), the expression for total energy consumption with SISO transmission, such that each stage in the network transmits to an immediate next stage in the network can be written as

$$
\begin{aligned}
& E_{\text {Total }}^{(1)}=\left\{\epsilon_{S I S O}\left(\frac{2^{b}-1}{b}\right) d^{2}+\frac{\left(P_{T}+P_{R}\right)}{B b}\right\} \\
& {\left[\frac{(I-2)(I+1) R}{2}+I R_{1}+R_{I}\right] .}
\end{aligned}
$$

where $\epsilon_{S I S O} \triangleq \frac{1}{6}(1+\alpha)\left(\frac{1}{p_{b}}\right) N_{0} G_{0}, R$ is defined as in Equation (19).

For the purpose of analysis in this subsection consider the following optimization problem:

$$
\begin{aligned}
& \underset{b}{\operatorname{minimize}} E_{\text {Total }}^{(1)}(b, a) \\
& \text { subject to } 2 \leq b \leq b_{\max }
\end{aligned}
$$

where $E_{\text {total }}^{(1)}$ is given by Equation (20) and $b_{\max }$ is determined by peak power value of the node.

We now state our first structural result as the following theorem. The theorem states that the optimal constellation size $b^{*}$ is an increasing function of correlation coefficient $a$.

Theorem 1: For a given number of stages $(I)$ in a network, spacing $(d)$, observation $S N R(\Gamma)$ the optimal constellation size $\left(b^{*}\right)$ of the optimization problem in Equation (21) is always an increasing function of the correlation coefficient (a). i.e.

$$
b^{*}(a) \triangleq \arg \min _{2 \leq b \leq b_{\max }} E_{\text {Total }}^{(1)}(b, a)
$$

is an increasing function of $a$.

Interpretation of Theorem 1: In general as the correlation coefficient increases, the sensor nodes can decrease their encoding rate and hence the amount of data transmitted decreases. This decrease in data implies shorter ON time for the sensor nodes and hence large constellation sizes can be deployed.

B. Structural Result on Optimal Number of Cooperating Nodes $\left(n^{*}\right)$ versus Correlation Coefficient $(a)$

For the case when $n$ nodes in each stage of the network cooperate to transmit from $k^{\text {th }}$ stage of the network to the $(k+1)^{\text {st }}$ stage $(k=1,2, \cdots, I)$ of the network, assuming each node in the network adopts a constellation size $b$ (i.e. $b_{i j}=b$ ), with stages from index $k=1,2, \cdots, I$ employing encoding rate of $R$ and encoding rate of $R_{I}$ by $I^{\text {th }}$ stage, the total energy consumption with MIMO transmission can be written as

$$
\begin{aligned}
& E_{\text {Total }}^{(2)}=\left\{\epsilon_{M I M O} n(K)^{\frac{1}{n}}\left(\frac{2^{b}-1}{b^{\frac{1}{n}+1}}\right) d^{2}+\frac{n\left(P_{T}+P_{R}\right)}{B b}\right. \\
& \left.+(n-1) E_{C-\text { local }}+E_{T x-\text { local }}\right\} \times \\
& {\left[\frac{(I-2)(I+1) R}{2}+I R_{1}+R_{I}\right] .}
\end{aligned}
$$

For the purpose of analysis in this subsection consider the following optimization problem

$$
\begin{aligned}
& \underset{n}{\operatorname{minimize}} E_{\text {Total }}^{(2)}(n, a) \\
& \text { subject to } 1 \leq n \leq N
\end{aligned}
$$


We now state our second structural result as the following theorem. The theorem proves that for arbitrarily low probability of error $p_{b}$, the optimal number of cooperating nodes $\left(n^{*}\right)$ is a decreasing function of the correlation coefficient $(a)$.

Theorem 2: For a given number of stages $(I)$ in the network, spacing $(d)$, constellation size (b), observation SNR $(\Gamma)$, the optimal number of cooperating nodes $\left(n^{*}\right)$ of the optimization problem in Equation (23) is a decreasing function of the correlation coefficient (a) for arbitrarily low probability of error $\left(p_{b}\right)$. i.e. as $p_{b} \rightarrow 0^{+}$

$$
n^{*}(a) \triangleq \arg \min _{1 \leq n} E_{\text {Total }}^{(2)}(n, a)
$$

is a decreasing function of correlation coefficient (a).

Interpretation of Theorem 2: The conclusion of Theorem 2 is interesting. The theorem suggests that for arbitrarily low probability of error $\left(p_{b}\right)$, when the data is correlated, the number of nodes participating in cooperative transmission can be less than the case when data is uncorrelated.

\section{Structural Result on Optimal rate $b^{*}$ versus Number of Cooperating Nodes $n$}

In this subsection we study as how the optimal rate $b^{*}$ varies with respect to the number of cooperating nodes $n$.

For analysis in this subsection we ignore circuit power consumption. Thus $E_{\text {Total }}^{(2)}$ in Equation (22) can be re-written as

$$
\begin{aligned}
& E_{\text {Total }}^{(3)}=[\epsilon_{M I M O} \underbrace{n(K)^{\frac{1}{n}}\left(\frac{2^{b}-1}{b^{\frac{1}{n}+1}}\right) d^{2}}+E_{T x-\text { local }}] \\
& {\left[\frac{(I-2)(I+1) R}{2}+I R_{1}+R_{I}\right]}
\end{aligned}
$$

where $\epsilon_{M I M O} \triangleq \frac{2}{3}(1+\alpha) N_{0} G_{0}\left(\frac{1}{r}\right)$ and $K \triangleq\left(\frac{4}{p_{b}}\right)$.

For the analysis in this subsection consider the following optimization problem:

$$
\begin{aligned}
& \text { minimize } E_{\text {Total }}^{(3)}(b, n) \\
& \text { subject to } 2 \leq b \leq b_{\max },
\end{aligned}
$$

where $E_{\text {Total }}^{(3)}$ is given by Equation (24).

We now state our final structural result as the following theorem. In this theorem we study as how the optimal constellation size $b^{*}$ varies with the number of cooperating nodes.

Theorem 3: For a given number of stages $(I)$ in a network, spacing $(d)$, correlation coefficient $(a)$ and observation SNR $(\Gamma)$ the optimal rate $\left(b^{*}\right)$ of the optimization problem in Equation (25) is a decreasing function of the number of cooperating nodes $(n)$. i.e.

$$
b^{*}(n) \triangleq \arg \min _{2 \leq b \leq b_{\max }} E_{\text {Total }}^{(2)}(n, b)
$$

is a decreasing function of $n$.

Remark 1: Since supermodularity doesn't differentiate between the two variables, hence from Theorem 3 it can be inferred that the optimal number of sensors $n^{*}$ participating in cooperative transmission to the sink, is a decreasing function of the rate $b$.

Interpretation of Theorem 3 and Remark 1: Theorem 3 and Remark 1 are intuitively appealing. Theorem 3 implies that owing to the cooperation among sensor nodes, the constellation size adopted by each sensor participating in cooperative transmission to minimize energy consumption decreases as the number of participating nodes increase.

While Remark 1 implies that as the constellation size adopted by each cooperating node increases, the number of cooperating nodes to minimize the energy consumption decreases.

\section{Numerical Results}

We assume a eleven stage network (i.e. $(I+1)=11$ ), with each stage separated by a distance of thirty metres (i.e. $d=30$ $\mathrm{m})$. The first 10 stages of the network denote data gathering stages, while the $11^{\text {th }}$ stage denotes the sink node. The system parameter details of $2.5 \mathrm{GHZ}$ radio in the Industrial-ScientificMedical (ISM) band are taken from [7]. The value of constant $(\alpha)$ is computed using the formula $\alpha=\frac{3}{\eta}\left(\frac{2^{\frac{c}{2}}-1}{2^{\frac{c}{2}}+1}\right)-1$, where $c$ denotes the constellation size and $\eta$ denotes drain efficiency of the amplifier whose value is provided in Table I. We assume each sensor node employs a constellation size equal to two (i.e. $b$ and $b_{\text {local }}$ are equal to two), hence $\alpha=1.85$. Finally, we assume $\left(\sigma^{2}\right)=0.01$ and $(\Delta)=0.001$.

We choose the probability of error $\left(p_{b}\right)=10^{-3}$. For the MIMO transmission case we assume the number of cooperating nodes in each stage as equal to two (i.e. $n_{i}=2$ for $i=1,2, \cdots, I$ ), hence the spatial rate is equal to one (i.e. $r=1)$. We assume the observation SNR is equal to twenty $\mathrm{dB}$ (i.e. $\Gamma=10 \mathrm{~dB}$ ). For a fixed value of constellation size and number of cooperating nodes, the optimization problem in equations (10) - (13) is a minimum cost network flow (MCNF) problem, hence the problem can be efficiently solved using the Matlog toolbox [9].

The plot of optimal energy consumption values versus the correlation coefficient is shown in Figure 1. As expected, the energy consumption value decreases with increase in value of correlation coefficient. It can be observed from the figure that when circuit energy is taken into account, then MIMO transmission may not be efficient. However, when transmission energy alone is taken into account then MIMO transmission is efficient. Also, when transmission energy alone is taken into account then the optimal energy consumption value obtained by solving MCNF problem coincides with the energy consumption value obtained via shortest path routing. However, when circuit energy consumption is taken into account then the energy consumption value obtained via shortest path routing is higher. From this, we can conclude that when transmission energy alone is considered then shortest path routing is optimal.

The plot of optimal energy consumption values versus the correlation coefficient for the case when probability of error $p_{b}=10^{-6}$ is shown in Figure 2. For the case when number 


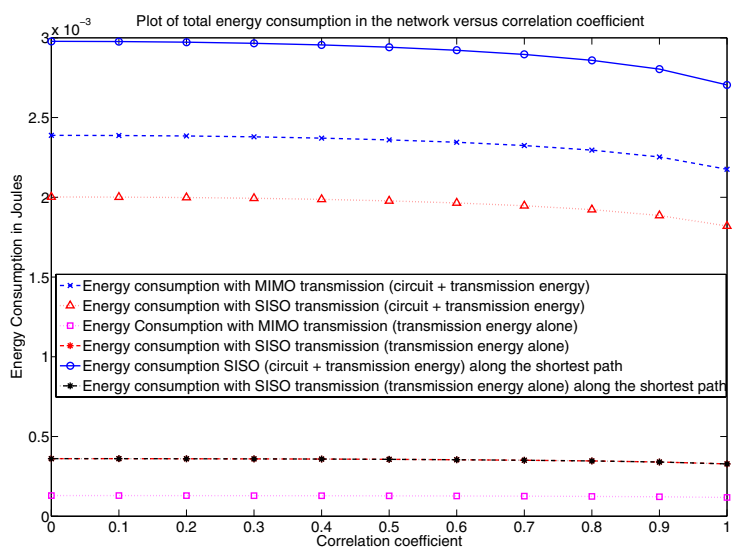

Fig. 1. The figure shows the plot of total energy consumption in the network versus the correlation coefficient. We fix the observation SNR $(\Gamma)$ equal to 10 $\mathrm{dB}$ and assume the desired probability of error $\left(p_{b}\right)$ equal to $10^{-3}$. Number of stages in the network are assumed equal to 11 . Distance between the various stages of the network is equal to 30 metres.

of cooperating nodes is equal to four, spatial rate $(r)$ is equal to $3 / 4$ as it is possible to construct an orthogonal STBC with this spatial rate as shown in [6], while for the case when the number of cooperating nodes is equal to eight, spatial rate $(r)$ is equal to $5 / 8$.

From Figure 2 it can be observed that when the desired probability of error is small MIMO transmission outperforms SISO transmission in terms of energy efficiency. For the given system parameter values, it has been found that a cluster with 4 cooperating nodes can be more energy efficient than compared to the case when the cluster has 2 and 8 cooperating nodes. In the former case, the diversity advantage provided by additional cooperating nodes is more than the increase in energy consumption due to local communication and use of STBC with spatial rate less than one. While in the latter case, the increase in energy consumption due to local communication and use of STBC with spatial rate less than one can be more than the diversity advantage provided by additional cooperating nodes. Hence, there exists an optimal number of cooperating nodes that can lead to high energy efficiency, which in the current case is equal to 4 .

\section{CONClusions}

We formulate minimum energy correlated data gathering in sensor networks based on cooperative MIMO techniques and DSC. We obtain important structural results on optimal constellation size and number of cooperating nodes. In particular, we prove that the optimal constellation size is an increasing function of correlation coefficient which implies the nodes can adopt higher constellation size when the data is correlated. For the MIMO case we prove that the optimal number of cooperating nodes is a decreasing function of correlation coefficient. This implies that for gathering correlated data the number of cooperating nodes can be less than that for gathering uncorrelated data. In our last structural

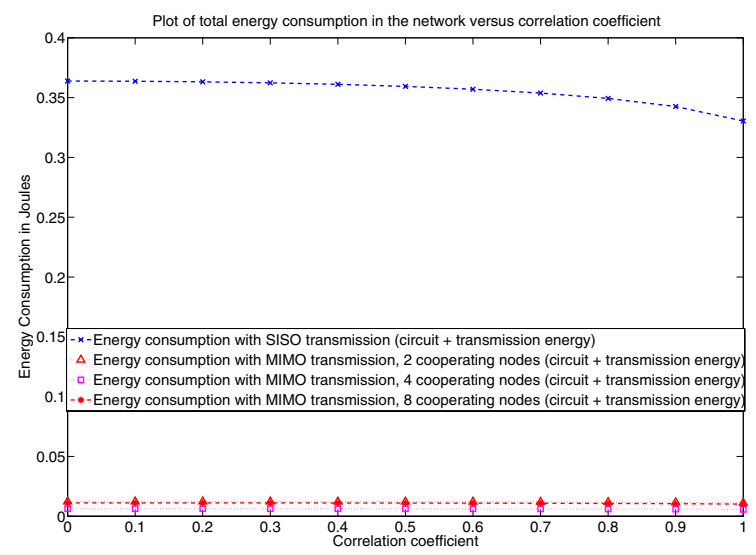

Fig. 2. The figure shows the plot of total energy consumption in the network versus the correlation coefficient. We fix the observation SNR $(\Gamma)$ equal to 10 $\mathrm{dB}$ and assume the desired probability of error $\left(p_{b}\right)$ equal to $10^{-6}$. Number of stages in the network are assumed equal to 11 . Distance between the various stages of the network is equal to 30 metres.

result we prove that the optimal constellation size adopted for cooperative transmission decreases with increase in number of cooperating nodes. Also, the optimal number of cooperating nodes decreases with increase in constellation size adopted for cooperative transmission. Finally from our numerical results, it has been observed that when desired probability of error is small, then MIMO transmission can be more energy efficient than SISO transmission. But for moderate values of probability of error SISO transmission can be more energy efficient. Furthermore, when circuit energy consumption of nodes is taken into account then shortest path routing may not be optimal in terms of minimizing total energy consumption in the network.

\section{REFERENCES}

[1] R. Cristescu, B. Beferull-Lozano, and M. Vetterli, "Networked SlepianWolf: Theory, Algorithms and Scaling Laws," IEEE Transactions on Information Theory, vol. 51, no. 12, pp. 4057-4073, Nov. 2005.

[2] R. Cristescu, B. Beferull-Lozano, M. Vetterli, and R. Wattenhofer, "Network Correlated Data Gathering With Explicit Communication: NPCompleteness and Algorithms," IEEE/ACM Transactions on Networking, vol. 14, no. 1, pp. 41-54, Feb. 2006.

[3] S. Cui and A. J. Goldsmith, "Cross-layer Design of Energy-constrained Networks Using Cooperative MIMO Techniques," EURASIP/Elsevier Signal Processing Journal, vol. 86, no. 8, pp. 1804-1814, Aug. 2006.

[4] D. M. Topkis, Supermodularity and Complementarity, Princeton University Press, New Jersey, 1998.

[5] L. S. Pillutla and V. Krishnamurthy, "Data Gathering and Fusion in Correlated Sensor Networks with Cooperative Transmission," IEEE Transactions on Signal Processing (submitted).

[6] V. Tarokh, H. Jafarkhani, and A. R. Calderbank, "Space-Time Block Codes from Orthogonal Designs," IEEE Transactions on Information Theory, vol. 45, no. 5, pp. 1456-1467, July 1999.

[7] S. Cui, A. J. Goldsmith, and A. Bahai, "Energy-efficiency of MIMO and Cooperative MIMO Techniques in Sensor Networks," IEEE Journal on Selected Areas in Communications, vol. 22, no. 6, pp. 1089-1098, Aug. 2004.

[8] B. D. O. Anderson and J. B. Moore, Optimal Filtering, Prentice-Hall, New Jersey, 1979.

[9] http://www.ie.ncsu.edu/kay/matlog/MatlogRef.htm, "Matlog: Logistics engineering matlab toolbox version 9 24-jan-2006," Matlog Reference. 